\title{
VARIAÇÃO DA MEDIDA TORÁCICA OBTIDA COM A FITA MÉTRICA TRADICIONAL COM FATOR DE CORREÇÃO E COM A FITA DE PESAGEM PARA BOVINOS
}

\author{
Bruno Almeida ABREU ${ }^{1}$, Chrissia Jardim MAGALHÃES ${ }^{1}$, Enrico DUAYER ${ }^{1}$, Sérgio Henrique Mattos \\ MACHADO $^{1} \&$ Denise Aparecida da SILVA ${ }^{1^{*}}$ \\ ${ }^{1}$ Universidade Iguaçu - UNIG, Campus V, Itaperuna, Rio de Janeiro, Brasil. \\ *Autor para correspondência: dearasp@yahoo.com.br \\ http://dx.doi.org/10.18571/acbm.084
}

\section{RESUMO}

Introdução: A estimativa do peso de bovinos pode ser realizada através do uso da fita torácica de pesagem ou com a fita métrica comum, devendo tal valor ser multiplicado por 2,8. O objetivo da pesquisa foi comparar os pesos obtidos com a fita métrica comum e o valor obtido através do uso da fita de pesagem desenvolvida especificamente para este fim em bovinos (fita torácica de pesagem). Material e métodos: A pesquisa foi realizada em São José do Calçado/ES em janeiro de 2015. No total foram estimados os pesos corporais de 20 fêmeas da raça Girolando com idade média de cinco meses com o uso da fita métrica comum e da fita torácica de pesagem para bovinos. Resultados: Os pesos corporais estimados através da fita métrica tradicional (X 2,8) variaram de 414,4 a $490 \mathrm{Kg}$ com média equivalente a 454,4 $\pm 19,5 \mathrm{Kg}$. Com o uso da fita torácica de pesagem os pesos corporais apresentaram valores que variaram de 271 a $424 \mathrm{Kg}$ com média igual a 346,7 $\pm 39,6 \mathrm{Kg}$. Os resultados apresentaram diferença significativa através da análise da variância com grau de confiança de $95 \%$, propondo-se um novo fator de correção equivalente a 2,12. Conclusão: o fator de correção 2,8 não corresponde ao índice ideal a ser utilizado em novilhas em se tratando do peso corporal estimado através do uso de fita métrica comum, sendo mais significativo em comparação à fita torácica de pesagem o índice equivalente a 2,12; tal índice apresenta melhor aplicação em animais mais jovens.

Palavras chave: medida torácica, fita métrica, fita de pesagem, bovinos.

\begin{abstract}
Introduction: The estimated weight of the cattle can be accomplished through the use of the weighing thoracic tape or with the traditional metric tape and such value is multiplied by 2.8 . The objective of the research was to compare the weights obtained with ordinary tape measure and the value obtained through the use of weighing tape specifically designed for this purpose in cattle (thoracic tape weighing). Material and Methods: The research was held in São José do Calçado/ES in January 2015. The body weights of 20 Gir breed females with an average age of five months were estimated by the use of common metric tape and of the thoracic tape weighing. Results: The estimated body weights through traditional metric tape (X 2.8) ranged from 414.4 to $490 \mathrm{~kg}$ with an average of $454.4 \pm 19.5 \mathrm{~kg}$. With the use of the thoracic tape weighing, the body weights showed values ranging $271-424 \mathrm{~kg}$ with a mean of $346.7 \pm 39.6 \mathrm{~kg}$. The results showed significant differences by analysis of variance with a degree of confidence of $95 \%$, proposing a new correction factor equivalent to 2.12. Conclusions: the correction factor 2.8 does not match the ideal index to be used on heifers in the case of body weight estimated by the use of ordinary tape metric, being more efficient the index 2,12 when compared to the thoracic tape weighing in cattle; this index shows improved application in younger animals.
\end{abstract}

Keywords: thoracic measure, metric tape, weighing tape, cattle. 


\section{Introdução}

No manejo normal dos animais bem como para a realização de exigências de nutrientes e sanidade, sua pesagem é de grande importância. No entanto, muitas propriedades não possuem balanças disponíveis para a pesagem dos animais e, muitas das vezes a opção é pela adoção de um método rápido e indireto que possa estimar o peso corporal. Além disso, há outros fatores que limitam o uso das balanças como, por exemplo, o estresse dos animais (SETIM et al., 2010). Sendo assim, uma forma de avaliação do peso corporal sem o uso de balanças é através de medidas corporais dos animais, sendo que várias são citadas na literatura com a finalidade de predizer o peso do animal, tais como o perímetro torácico, o comprimento corporal, a altura da cernelha e da garupa e o comprimento da garupa. Apesar de existirem discrepâncias sobre qual medida individual é a mais eficaz, a acurácia da predição tem sido geralmente alta especialmente quando mais de uma medida é considerada (HEIRINCHS et al., 1992; KHALIL; VACCARO, 2002). Alguns estudos demonstram que há uma elevada correlação entre o peso vivo (PV) do animal e determinadas medidas corporais, podendo algumas destas ser utilizadas com o intuito de estimar o PV dos animais na ausência de balança para pesagem (SOUZA et al., 2007).

Estudos realizados em ovinos revelaram que as equações geradas a partir das medidas biométricas são eficazes para estimar o peso vivo e compacidade corporal de animais de corte, machos e fêmeas, de diferentes raças e idades. Os autores concluíram, ainda, que os gráficos de dispersão são altamente representativos para interpretar o comportamento biológico de um rebanho e fornecem subsídios para o aperfeiçoamento das práticas de manejo e controle zootécnico (SOUZA et al., 2009). E, ainda, estudos revelam as relações entre medidas corporais e características de desempenho e correlações genéticas altas entre perímetro torácico, largura da garupa e peso tomado à mesma idade (CYRILLO et al., 2013).

No caso do perímetro torácico é avaliado o comprimento corporal correspondente a uma linha reta entre a articulação escápulo-umeral e a tuberosidade coxal do ílio, o comprimento da garupa equivale a uma linha reta entre a tuberosidade coxal do ílio e a tuberosidade isquiática, e a altura da garupa corresponde à distância entre o solo e a tuberosidade sacral do ílio com os animais em superfície plana (REIS et al., 2008).

Um método indireto de pesagem de animais, de baixo custo, prático e menos estressante consiste na medida da circunferência do tórax do animal, através da fita torácica de pesagem, a qual foi desenvolvida a partir de equações de estimativa do peso pelo perímetro torácico de um total de 5.723 novilhas em fazendas comerciais na Pensilvânia - EUA (SETIM et al., 2010). Segundo Silva et al. (2006) a medida torácica apresenta alta correlação com o peso vivo do animal, sendo assim de escolha para a estimativa de seu peso.

Cada parte do corpo cresce a uma taxa diferente (alometria), considerando-se que cada tecido apresenta seu próprio objetivo de crescimento, função e resposta às limitações nutricionais bem como alterações ambientais (DI MARCO et al., 2007), o que pode justificar a melhor correlação do perímetro torácico com o peso corporal.

Como se trata de uma estimativa, vários estudos já foram realizados com o intuito de avaliar a eficácia e consequente credibilidade da fita torácica de pesagem. Ao comparar os valores do peso de animais pesados em balança mecânica com aqueles obtidos nos mesmos animais com a fita torácica, Setim et al. (2010) verificaram diferenças que variaram de 5,0 a 12 $\mathrm{Kg}$ superiores para a medida com a fita torácica, bem como de 1,0 a 8,0 Kg inferiores para a fita. Os valores inferiores ocorreram apenas em machos, enquanto os valores superiores para a fita foram observados principalmente em fêmeas. Apesar das variações, os autores concluíram que a fita torácica de pesagem é uma excelente ferramenta para obtenção do peso dos animais, em especial em pequenas propriedades rurais, devido à inviabilidade de aquisição de uma balança mecânica ou digital, as quais apresentam um alto custo. 


\section{Biomédica Brasiliensia}

Vale salientar que as correlações entre a medida em centímetros obtida a partir da fita métrica tradicional e a fita torácica de pesagem são realizadas através de regressões e podem ser influenciadas por fatores como a raça, idade, condição corporal e estado fisiológico do animal (HEINRICHS et al., 1992). As fitas torácicas de pesagem disponibilizadas no comércio são largamente utilizadas atualmente em todas as raças animais e sob quaisquer condições ambientais, portanto com resultados que podem ser discrepantes do peso real dos animais. Porém, um problema ainda maior pode ocorrer ao se estimar o peso dos animais, pois apesar do baixo custo da fita torácica de pesagem comparativamente ao custo da balança mecânica ou digital, ainda há proprietários que se utilizam da fita métrica tradicional (de costura, fita métrica comum), quando então o valor encontrado é multiplicado por um fator de correção, o qual, geralmente, é citado como equivalente a 2,8. Tal medida parece ser de cunho popular, pois não se encontraram na literatura quaisquer estudos específicos que possam corroborar tal correlação. Em se tratando do peso corporal para a comercialização do animal, não haverá problemas uma vez que os animais serão devidamente pesados em balança mecânica ou digital nos abatedouros. No entanto, ao se realizar cálculos referentes a nutrientes e, principalmente, para administração de medicamentos, as diferenças no peso corporal podem levar a quadros de subdosagem ou superdosagem e, neste caso colocar em risco a vida do animal, principalmente em se tratando de medicamentos que apresentam baixo índice terapêutico e, portanto, alto índice de toxicidade.

$\mathrm{O}$ objetivo da pesquisa foi verificar se há diferença significativa entre os pesos obtidos à partir do perímetro torácico com a fita métrica comum (cujo valor em centímetros é multiplicado por 2,8 ) e o valor obtido através do uso da fita de pesagem desenvolvida especificamente para este fim em bovinos (fita torácica de pesagem).

\section{Material e Métodos}

A pesquisa foi realizada na propriedade Agropecuária Vale do Sapecado, localizada no município de São José do Calçado/ES no mês de janeiro de 2015. No total foram avaliados os pesos corporais de 20 fêmeas da raça Girolando com idade média de cinco meses e mantidas sob o mesmo sistema de criação (pastagem, alimentação e água ad libitum) e no mesmo pasto, separadas dos demais animais da propriedade. O peso foi avaliado através de dois métodos diferentes: (1) com o uso da fita métrica tradicional (comum), sendo o resultado obtido em centímetros multiplicado por 2,8 (partindo-se de informações de que cada centímetro corporal pesa em média $2,8 \mathrm{Kg}$.) e (2) com o uso da fita torácica de pesagem para bovinos, da marca Bovitec ${ }^{\circledR}$ (figura 1). A medida do perímetro torácico foi realizada caudalmente à escápula passando pelo esterno e pelos processos espinhais das vértebras torácicas, através do uso da fita métrica tradicional e da fita torácica de pesagem, conforme figura 2.

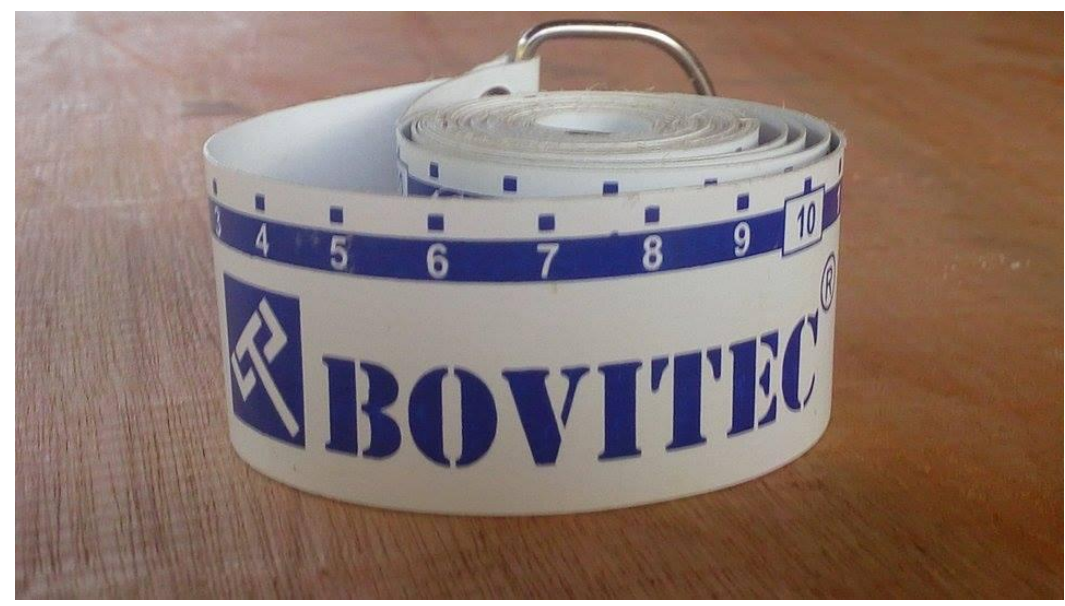

Figura 1. Fita torácica de pesagem para bovinos (Bovitec®) 


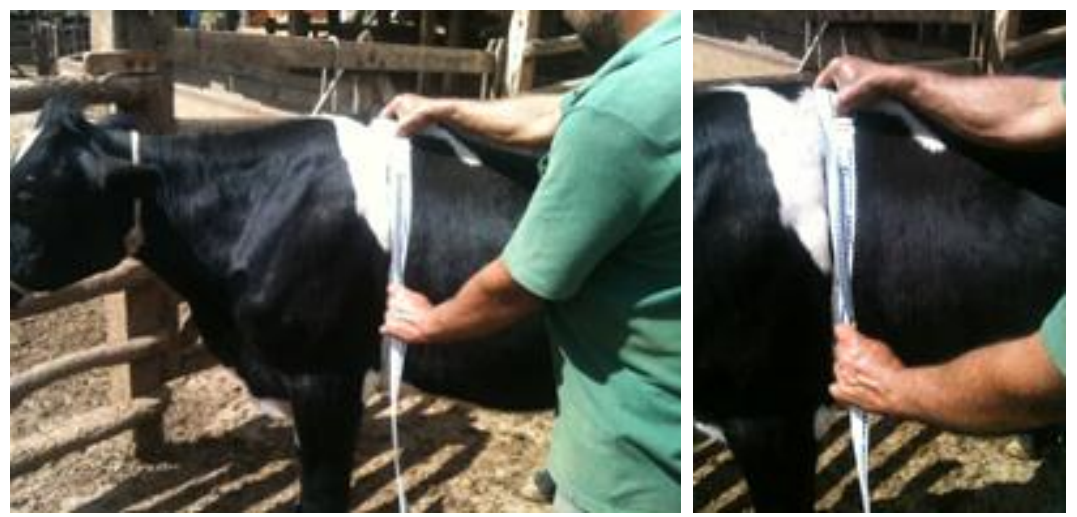

Figura 2. Medida do perímetro torácico. Fonte: Maia (2013).

Os resultados foram avaliados através da análise de variância (ANOVA) considerando-se o nível de significância de 0,05 (GOMES, 2000).

\section{Resultados e Discussão}

Os pesos corporais estimados através da fita métrica tradicional (após a multiplicação por 2,8) variaram de 414,4 a $490 \mathrm{Kg}$ com média equivalente a 454,4 $\pm 19,5 \mathrm{Kg}$. Com o uso da fita torácica de pesagem os pesos corporais apresentaram valores que variaram de 271 a $424 \mathrm{Kg}$ com média igual a $346,7 \pm 39,6 \mathrm{Kg}$. (Gráfico 1). Sendo assim, houve uma redução de $34,6 \%$ no peso corporal da fêmea com menor peso e de $13,5 \%$ da fêmea com maior peso corporal com o uso da fita torácica de pesagem.

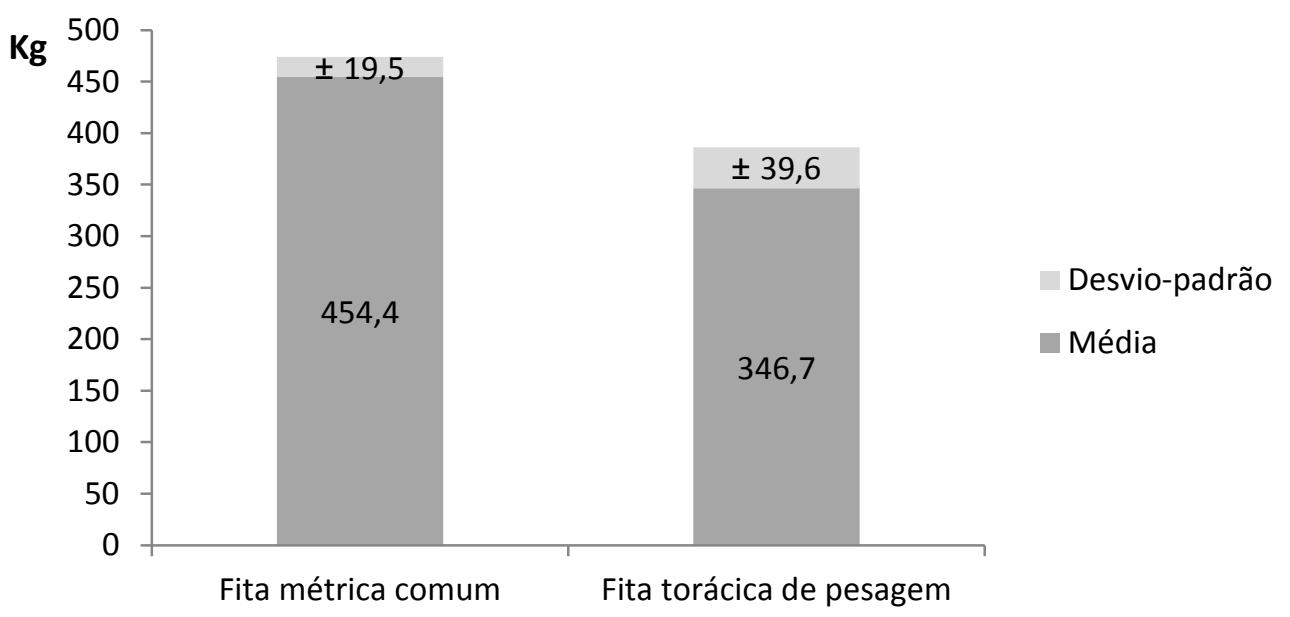

Gráfico 1: Média do peso corporal de fêmeas de bovinos com o uso da fita métrica comum (valor obtido multiplicado por 2,8 ) e através do uso da fita torácica de pesagem.

Os pesos corporais obtidos individualmente com o uso da fita métrica tradicional (multiplicado por 2,8) e com a fita torácica de pesagem podem ser melhor observados no gráfico 2.

Ao se aplicar o tratamento estatístico, os resultados apresentaram diferença significativa através da análise da variância considerando-se o grau de confiança de $95 \%$, ou seja, o peso estimado com o uso do valor de correção 2,8 é significativamente diferente do peso obtido através da fita torácica de pesagem $((\mathrm{F}(1,38)=118,83 ; \alpha=0,05)$. Portanto, o fator 2,8 não se 
mostrou aplicável para a amostra analisada e, assim, um novo fator de correção equivalente a 2,12024 é proposto (ao menos, em se tratando de novilhas).

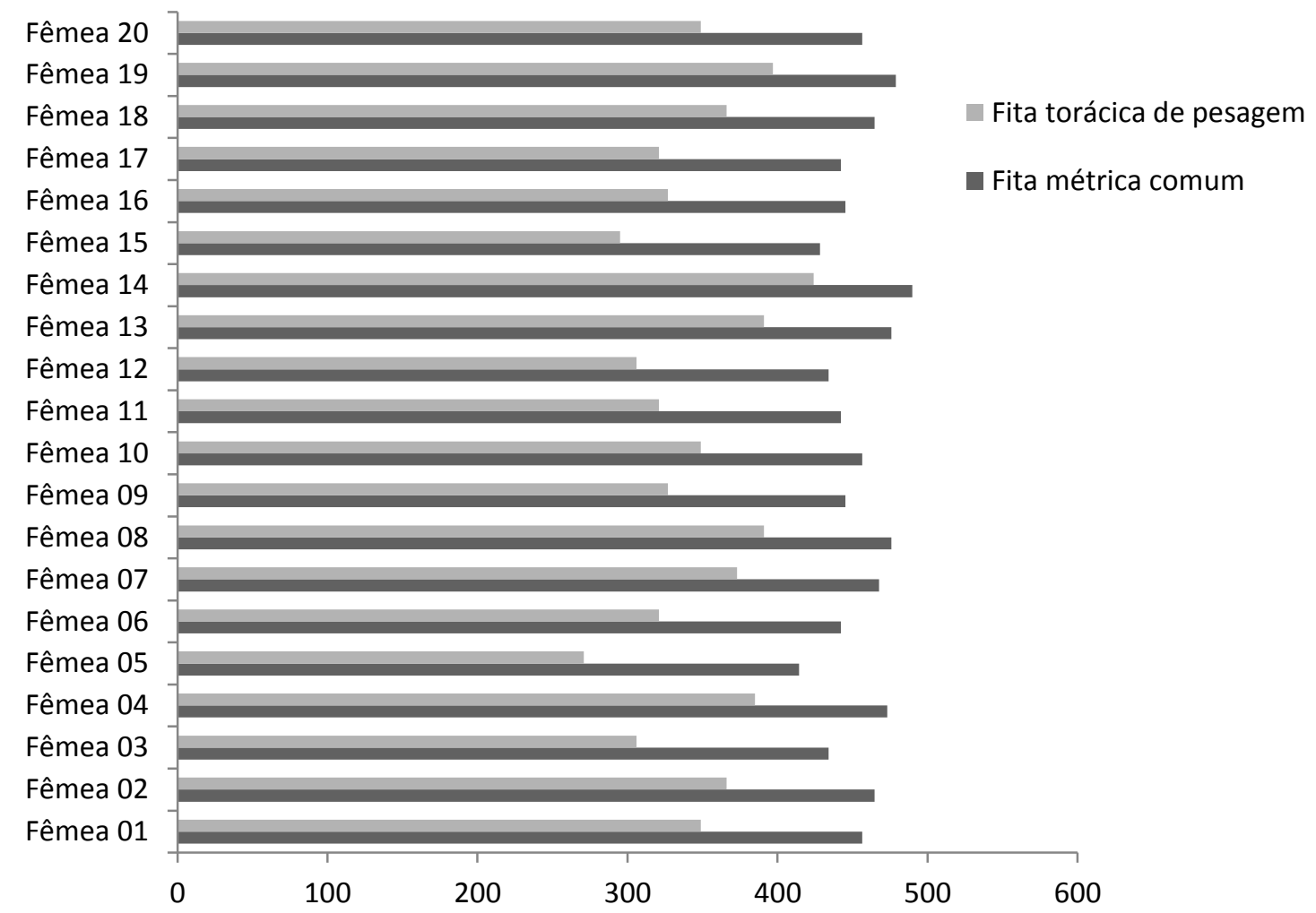

Gráfico 2: Valores individuais do peso corporal de fêmeas de bovinos com o uso da fita métrica comum (valor obtido multiplicado por 2,8) e através do uso da fita torácica de pesagem.

Os pesos corporais estimados através da fita métrica tradicional (após a multiplicação pelo novo índice $=2,12$ ) variaram de 313,8 a $371 \mathrm{Kg}$ com média equivalente a $344,1 \pm 14,7 \mathrm{Kg}$. Sendo assim, agora considerando-se o novo índice proposto, houve uma redução de $13,6 \%$ no peso corporal da fêmea com menor peso e aumento de $14,3 \%$ da fêmea com maior peso corporal com o uso da fita torácica de pesagem.

Foi possível observar maior diferença entre os valores obtidos através das duas fitas em relação aos animais de maior e menor peso, no entanto, tais variações são menores com o novo valor de correção proposto o qual demonstrou valores mais próximos nos animais de menor peso, justamente aqueles mais susceptíveis a quadros de intoxicação em quadros de superdosagem de medicamentos. No caso de animais de maior peso foi observado o aumento nos valores do peso corporal ao se comparar o novo índice proposto e a fita torácica de pesagem, indicando, portanto, a melhor indicação do índice proposto para animais mais jovens e de menor peso. Abaixo (gráfico 3) pode-se observar os valores obtidos com o novo índice de correção proposto, podendo-se comparar ao gráfico 2, referente ao índice de 2,8. 


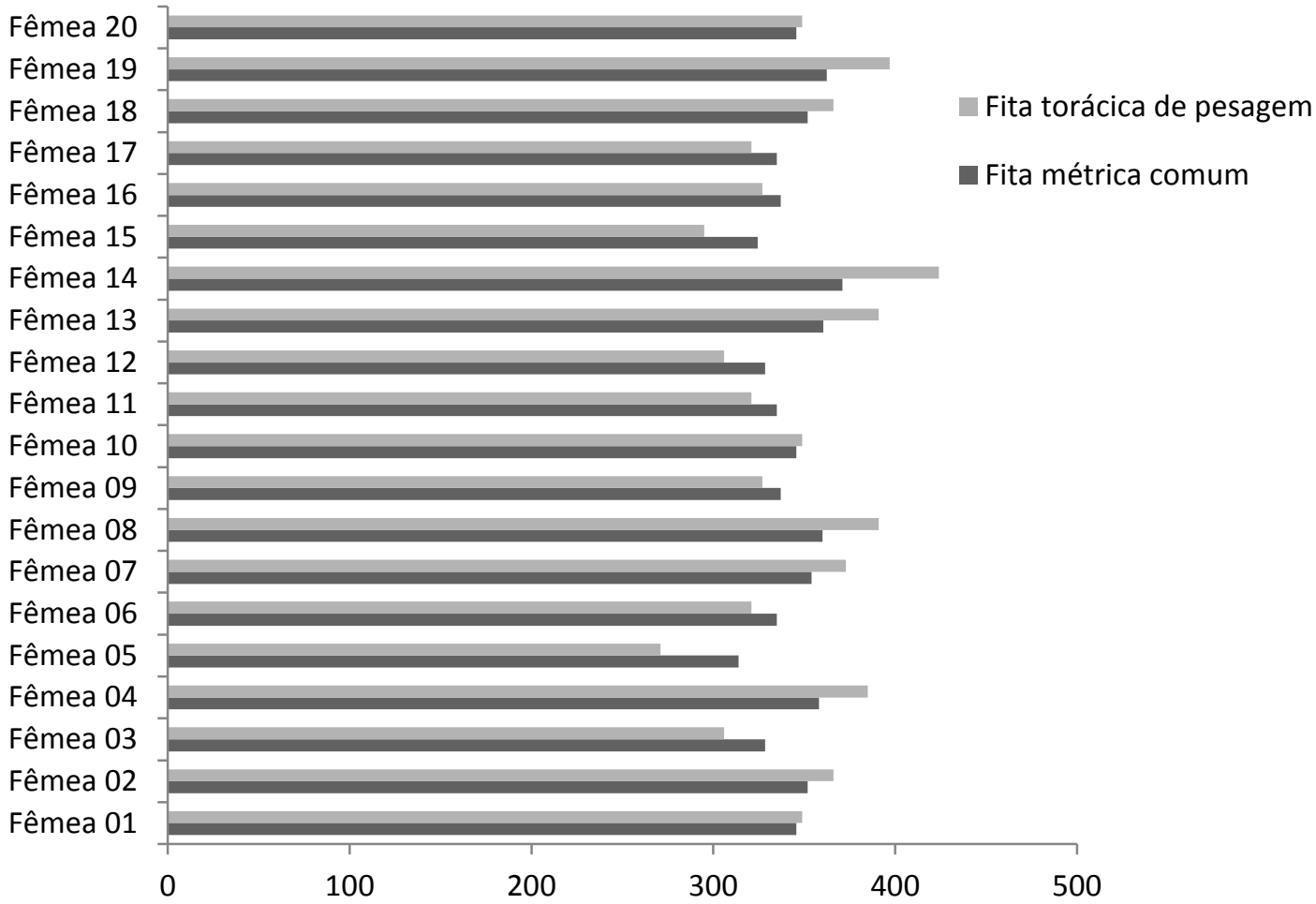

Gráfico 3. Valores individuais do peso corporal de fêmeas de bovinos com o uso da fita métrica comum (valor obtido multiplicado pelo novo índice proposto $=2,12$ ) e através do uso da fita torácica de pesagem.

No dia-a-dia, nas propriedades de menor área, o peso dos animais visando a realização dos diferentes manejos, tais como a administração de medicamentos, é obtido através de estimativa visual que, provavelmente, leva a erros ainda superiores aos observados com a fita métrica tradicional (de costura). A proposta de um novo índice de correção equivalente a 2,12 pode ser uma alternativa a ser implantada nestas propriedades a fim de minimizar os erros de peso estimado e garantir maior segurança, em especial no cálculo de dose de medicamentos.

\section{Conclusões}

De acordo com a metodologia utilizada pode-se concluir que: o fator de correção 2,8 não corresponde ao índice ideal a ser utilizado em novilhas em se tratando do peso corporal estimado através do uso de fita métrica comum, sendo mais significativo em comparação à fita torácica de pesagem o índice equivalente a 2,12; tal índice apresenta melhor aplicação em animais mais jovens e, portanto, com menor peso corporal.

\section{Bibliografia}

CYRILLO, J.N.S.G.; MERCADANTE, M.E.Z.; BONILHA, S.F.M. Relações entre medidas biométricas, características de carcaça e cortes cárneos comerciais em bovinos Zebu e Caracu. CAPTA; APTA; PRDTA. Artigos Técnicos, 2013. Disponível em: http://pt.engormix.com/MApecuaria-corte/frigorifico/artigos/relacoes-entre-medidas-biometricas-t1523/378-p0.htm Acesso em 04 de abr. 2105.

DI MARCO, O.N.; BARCELOS, J.O.J.; COSTA, E.C. Crescimento de bovinos de corte. Porto Alegre: Departamento de Zootecnia da Universidade Federal do Rio Grande do Sul. Núcleo de Estudos em Sistemas de Produção de Bovinos de Corte e Cadeia Produtiva, 2007, 278 p. 
GOMES, F.P. Curso de estatística experimental. Piracicaba: Universidade de São Paulo, 14. ed. , 477 p., 2000.

HEINRICHS, A.J. et al. Predicting body weight and wither height in Holstein heifers using body measurements. Journal of Dairy Science, v. 75, p. 3576-3581, 1992.

KHALIL, R.; VACCARO, L. Body weights and measurements in dual purpose cows: their interrelation and association with genetic merit for three production traits. Zootecnia Tropical, v. 20, p. 11-30, 2002.

MAIA, P.V. Monitoramento do ganho de peso diário em bezerras e novilhas. Rehagro, Artigos Técnicos, 2013. Disponível em http://rehagro.com.br/plus/modulos/noticias/ler.php? cdnoticia=2605 Acesso em 12 de junho 2015.

REIS, G.L.; ALBUQUERQUE, F.H.M.A.R.; VALENTE, B.D. et al. Predição do peso vivo a partir de medidas corporais em animais mestiços Holandês/Gir. Cienc. Rural, v. 38, n. 3, p. 778783, 2008.

SETIM, D.H.; VANZAN, M.; FERNANDES, M.V. et al. Comprovação da eficácia do uso da fita torácica de pesagem em bovinos leiteiros. 2010. Disponível em: http://www.cafw.ufsm.br/mostraciencias/2011/resumos/210.pdf Acesso em 04 de abr. 2015.

SILVA, D.C.; AZEVEDO, D.M.M.R.; ALVES, A.A. et al. Estimativa do peso vivo através do perímetro torácico de ovinos Santa Inês. Revista Científica Produção Animal, v. 8, n. 2, 2006.

SOUZA, S.F.; COSTA, R.G.; RESENDE, K.T. et al. Avaliação das curvas de crescimento de caprinos das raças Saanen e 7/8 Boer durante a fase de aleitamento. Mem. Reunião Anual da Sociedade Brasileira de Zootecnia, v. 44, n. 127 (Resumo), 2007.

SOUZA, S.; LEAL, A.; BARIONI, C. et al. Utilização de medidas biométricas para estimar peso vivo em ovinos. Empresa Brasileira de Pesquisa Agropecuária, Embrapa Tabuleiros Costeiros, Aracaju, Asociación Latinoamericana de Producción Animal, v. 17, n. 3-4, p. 61-66, 2009. 